\title{
AN INVARIANT RIEMANN TYPE INTEGRAL DEFINED BY FIGURES
}

\author{
ALEXEI NOVIKOV AND WASHEK F. PFEFFER
}

(Communicated by Andrew M. Bruckner)

\begin{abstract}
We show that a multidimensional generalized Riemann integral defined by means of rectangular figures is already invariant with respect to lipeomorphic changes of coordinates.
\end{abstract}

In recent years, following the work of Henstock, Kurzweil, and Mawhin, several authors proposed definitions of a generalized Riemann integral that can be used to establish the Stokes theorem for noncontinuously differentiable forms. All these definitions are based on concepts which are a priori invariant with respect to changes of coordinates, e.g., sets of finite perimeter [P1, P2] or partitions of unity [JK1, JK2, KMP]. In this note we show that an integral defined by means of figures, i.e., finite unions of intervals, is already invartiant with respect to lipeomorphisms.

\section{Preliminaries}

Throughout this note, $m \geq 1$ is a fixed integer, and the metric in $\mathbf{R}^{m}$ is induced by the norm $|x|=\max \left\{\left|\xi_{1}\right|, \ldots,\left|\xi_{m}\right|\right\}$. If $E \subset \mathbf{R}^{m}$, then $d(E), E^{-}$, $E^{\circ}$, and $\partial E$ denote, respectively, the diameter, closure, interior, and boundary of $E$. In $\mathbf{R}^{m}$ we consider the Lebesgue measure $\lambda$ and the $(m-1)$-dimensional Hausdorff measure $\mathscr{H}$. We say that the sets $A, B \subset \mathbf{R}^{m}$ overlap whenever $\lambda(A \cap B)>0$. A set $T \subset \mathbf{R}^{m}$ is called thin if it is of $\sigma$-finite measure $\mathscr{H}$.

DeGiorgi's perimeter, finite or infinite, of a bounded Borel set $E \subset \mathbf{R}^{m}$ is denoted by $\|E\|$ (see [Z, Definition 5.4.1]). By $\mathscr{K}$ we denote the family of all compact sets $K \subset \mathbf{R}^{m}$ with $\mathscr{H}(\partial K)<+\infty$. According to [Z, Theorem 5.8.5], $\|K\|<\mathscr{H}(\partial K)$ for each $K \in \mathscr{K}$.

An interval is always a compact nondegenerate subinterval of $\mathbf{R}^{m}$, i.e., the product $C=\prod_{j=1}^{m}\left[a_{j}, b_{j}\right]$ where $a_{j}<b_{j}$ are real numbers; when $b_{j}-a_{j}=d$ for $j=1, \ldots, m$, we say that $C$ is a cube of diameter $d(C)=d$. A figure is a finite, possibly empty, union of intervals. Each figure $A$ belongs to $\mathscr{K}$, and $\|A\|=\mathscr{C}(\partial A)$. If $A$ and $B$ are figures, then so are the sets $A \cup B$ and $(A-B)^{-}$. The number

$$
r(A)=\frac{\lambda(A)}{d(A)\|A\|}
$$

Received by the editors June 29, 1992.

1991 Mathematics Subject Classification. Primary 26A39, 26B15. 
is called the regularity of a nonempty figure $A$.

All functions we consider are real-valued. A nonnegative function $\delta$ on a set $E \subset \mathbf{R}^{m}$ is called a gage on $E$ whenever the set $\{x \in E: \delta(x)=0\}$ is thin. A caliber is a sequence $\left\{\eta_{j}\right\}$ of positive real numbers.

A partition in a figure $A$ is a collection $P=\left\{\left(A_{1}, x_{1}\right), \ldots,\left(A_{p}, x_{p}\right)\right\}$, possibly empty, where $A_{1}, \ldots, A_{p}$ are nonoverlapping subfigures of $A$ and $x_{i} \in A_{i}$ for $i=1, \ldots, p$. Given $\varepsilon>0$, a gage $\delta$ on $A$, and a caliber $\eta=\left\{\eta_{j}\right\}$, we say that $P$ is

(1) $\varepsilon$-regular if $r\left(A_{i}\right)>\varepsilon$ for $i=1, \ldots, p$;

(2) $\delta$-fine if $d\left(A_{i}\right)<\delta\left(x_{i}\right)$ for $i=1, \ldots, p$;

(3) $(\varepsilon, \eta)$-approximating if $\left(A-\bigcup_{i=1}^{p} A_{i}\right)^{-}=\bigcup_{j=1}^{k} B_{j}$ where $B_{1}, \ldots, B_{k}$ are nonoverlapping figures with $\left\|B_{j}\right\|<1 / \varepsilon$ and $\lambda\left(B_{j}\right)<\eta_{j}$ for $j=$ $1, \ldots, k$.

Lemma 1.1. Let $\delta$ be a gage in a figure $A$. Then for each positive $\varepsilon<1 /(2 m)$ and each caliber $\eta$ there is a $\delta$-fine $\varepsilon$-regular and $(\varepsilon, \eta)$-approximating partition in $A$.

This existence result has been proved in [P3, §4]. We shall also need a lemma, which follows from [Fa, Theorem 5.1].

Lemma 1.2. There is a constant $\kappa$, which depends only on $m$, and has the following property: if $E \subset \mathbf{R}^{m}$ and $\mathscr{H}(E)<a$, then for each $\eta>0$ we can find a finite or infinite sequence of cubes $\left\{C_{n}\right\}$ of diameters less than $\eta$ and such that $E \subset \bigcup_{n} C_{n}^{\circ}$ and $\sum_{n}\left\|C_{n}\right\|<\kappa a$.

\section{THE INTEGRAL}

Definition 2.1. A function $f$ defined on a figure $A$ is called integrable in $A$ if there is a real number $I$ having the following property: given $\varepsilon>0$, we can find a gage $\delta$ on $A$ and a caliber $\eta$ so that

$$
\left|\sum_{i=1}^{p} f\left(x_{i}\right) \lambda\left(A_{i}\right)-I\right|<\varepsilon
$$

for each $\delta$-fine $\varepsilon$-regular and $(\varepsilon, \eta)$-approximating partition $\left\{\left(A_{1}, x_{1}\right), \ldots\right.$, $\left.\left(A_{p}, x_{p}\right)\right\}$ in $A$.

It follows from Lemma 1.1 that the number $I$ of Definition 2.1 is determined uniquely by the integrable function $f$. We call it the integral of $f$ over $A$ and denote it by $\int_{A} f$.

The basic properiies of the integral are established in [P3, $\S \S 6$ and 7]. For the reader's convenience we restate the main results needed for the change of variables theorem (Theorem 3.3).

A function $F$ defined on a family $\mathscr{F}$ of bounded Borel sets is called:

(1) additive if $F(A)=\sum_{D \in \mathscr{D}} F(D)$ for each set $A \in \mathscr{F}$ and each nonoverlapping family $\mathscr{D} \subset \mathscr{F}$ with $\cup \mathscr{D}=A$;

(2) continuous if, given $\varepsilon>0$, there is an $\eta>0$ such that $|F(A)|<\varepsilon$ for each $A \in \mathscr{F}$ with $\|A\|<1 / \varepsilon$ and $\lambda(A)<\eta$. 
Proposition 2.2. Let $f$ be an integrable function in a figure $A$. Then $f$ is integrable in each figure $B \subset A$ and the map $F: B \mapsto \int_{B} f$ is an additive continuous function.

Proposition 2.3. Let $f$ be a function on a figure $A$. Then $f$ is integrable in $A$ if and only if there is an additive continuous function $F$ defined on all subfigures of $A$ and having the following property: given $\varepsilon>0$, we can find a gage $\delta$ on $A$ so that

$$
\sum_{i=1}^{p}\left|f\left(x_{i}\right) \lambda\left(A_{i}\right)-F\left(A_{i}\right)\right|<\varepsilon
$$

for each $\delta$-fine $\varepsilon$-regular partition $\left\{\left(A_{1}, x_{1}\right), \ldots,\left(A_{p}, x_{p}\right)\right\}$ in $A$.

Proposition 2.2 is identical to [P3, Proposition 6.4]. Using the additivity and continuity of $F$, it is easy to show that the condition of Proposition 2.3 is sufficient; that it is also necessary follows from Henstock's lemma [P3, Proposition 6.5]. The function $F$ of Proposition 2.2 coincides with that of Proposition 2.3, and we call it the indefinite integral of $f$ in $A$.

\section{THE INVARIANCE}

Lemma 3.1. Let $K \in \mathscr{K}$ and $\mathscr{H}(\partial K)<a$. There is a sequence $\left\{A_{n}\right\}$ of figures such that $A_{n} \subset K^{\circ}$ and $\left\|A_{n}\right\|<\kappa a$, for $n=1,2, \ldots$, and $\lim \lambda\left(K-A_{n}\right)=0$.

Proof. Since $\partial K$ is compact, it follows from Lemma 1.2 that for each integer $n \geq 1$ there are cubes $C_{1}, \ldots, C_{k}$ of diameters less than $1 / n$ and such that $\partial K \subset \bigcup_{i=1}^{k} C_{i}^{\circ}$ and $\sum_{i=1}^{k}\left\|C_{i}\right\|<\kappa a$. Then $A_{n}=\left(K-\bigcup_{i=1}^{k} C_{i}\right)^{-}$is a subfigure of $K^{\circ}$ with $\left\|A_{n}\right\|<\kappa a$ and

$$
\lambda\left(K-A_{n}\right) \leq \sum_{i=1}^{k} \lambda\left(C_{i}\right) \leq \frac{1}{n} \cdot \frac{1}{2 m} \sum_{i=1}^{k}\left\|C_{i}\right\|<\frac{1}{n} \cdot \frac{\kappa a}{2 m} .
$$

Proposition 3.2. Let $F$ be an additive continuous function defined on all subfigures of a figure $A$. Then $F$ has a unique additive continuous extension, still denoted by $F$, to the family $\{K \in \mathscr{K}: K \subset A\}$.

Proof. On the family $\mathscr{B}$ of all Borel subsets of $A$ we consider a metric

$$
\rho(B, C)=\lambda[(B-C) \cup(C-B)]
$$

and denote by $\operatorname{cl} \mathscr{C}$ the $\rho$-closure of a family $\mathscr{C} \subset \mathscr{B}$. According to $[\mathrm{Z}$, Corollary 5.3.4], for $n=1,2, \ldots$, the metric $\rho$ is complete on the family $\mathscr{B}_{n}=\{B \in \mathscr{B}:\|B\| \leq n\}$. Since

$$
\begin{aligned}
|F(B)-F(C)| & =\left|F\left[(B-C)^{-}\right]-F\left[(C-B)^{-}\right]\right| \\
& \leq\left|F\left[(B-C)^{-}\right]\right|+\left|F\left[(C-B)^{-}\right]\right|
\end{aligned}
$$

for all figures $B, C \subset A$, the function $F$ is uniformly $\rho$-continuous on the family $\mathscr{F}_{n}$ of all figures contained in $\mathscr{B}_{n}$. Consequently, $F$ has a unique $\rho$ continuous extension to cl $\mathscr{F}_{n}$. As $\left\{\mathscr{F}_{n}\right\}$ is an increasing sequence, $F$ has an additional unique extension to $\mathscr{F}=\bigcup_{n=1}^{\infty} \mathrm{cl} \mathscr{F}_{n}$, still denoted by $F$. It is easy to verify that $F$ is additive and continuous on $\mathscr{F}$. As $\mathscr{K} \subset \mathscr{F}$ by Lemma 3.1, the proposition is proved. 
Let $E \subset \mathbf{R}^{m}$ be a measurable set. For a Lipschitz map $\Phi: E \rightarrow \mathbf{R}^{m}$, we denote by $\operatorname{det} \Phi$ the determinant of the differential $D \Phi$ of $\Phi$. By the Kirszbraun and Rademacher theorems [Fe, Theorems 2.10 .43 and 3.1.6] the function $\operatorname{det} \Phi$ is defined almost everywhere in $E$, and by [P2, Lemma 5.16] it is determined uniquely by $\Phi$ up to a set of measure zero. A Lipschitz map $\Phi: E \rightarrow \mathbf{R}^{m}$ is called a lipeomorphism if it is injective and the inverse map $\Phi^{-1}: \Phi(E) \rightarrow \mathbf{R}^{m}$ is also Lipschitz.

Theorem 3.3. Let $\Phi: A \rightarrow B$ be a lipeomorphism from a figure $A$ onto a figure $B$, and let $f$ be an integrable function on $B$. Then $f \circ \Phi \cdot|\operatorname{det} \Phi|$ is integrable on $A$ and

$$
\int_{A} f \circ \Phi \cdot|\operatorname{det} \Phi|=\int_{B} f .
$$

Proof. There are positive constants $\alpha$ and $\beta$ such that

$$
\alpha\left|x-x^{*}\right| \leq\left|\Phi(x)-\Phi\left(x^{*}\right)\right| \leq \beta\left|x-x^{*}\right|
$$

for all $x, x^{*} \in A$. If $C$ is a subfigure of $A$, then it follows from [Fa, Lemma 1.8] that $\Phi(C)$ belongs to $\mathscr{K}$ and satisfies the inequalities

$$
\lambda[\Phi(C)] \geq \alpha^{m} \lambda(C) \text { and }\|\Phi(C)\| \leq \mathscr{H}[\partial \Phi(C)] \leq \beta^{m-1}\|C\| .
$$

By Propositions 2.2 and 3.2, the indefinite integral of $f$ in $B$ has a unique additive and continuous extension $F$ to the family $\{K \in \mathscr{K}: K \subset B\}$. In view of the above inequalities, the map $G: C \mapsto F[\Phi(C)]$ is an additive continuous function defined on all subfigures of $A$. We use Proposition 2.3 to show that $G$ is an indefinite integral of $f \circ \Phi \cdot|\operatorname{det} \Phi|$ in $A$.

To this end, choose an $\varepsilon>0$, and use [R, Theorem 7.2.4] to find a $\lambda$-zero set $N \subset A$ and a positive function $\Delta$ on $A$ so that

$$
|f \circ \Phi(x)| \cdot|\operatorname{det} \Phi(x) \lambda(C)-\lambda[\Phi(C)]|<\varepsilon \lambda(C)
$$

for each $x \in A-N$ and each figure $C \subset A$ with $x \in C, r(C)>\varepsilon$, and $d(C)<\Delta(x)$. Since $\lambda[\Phi(N)]=0$, by [P3, Corollary 6.8], we may assume that $f(y)=0$ for each $y \in \Phi(N)$ and, consequently, that the previous inequality holds for all $x \in A$.

By Proposition 2.3, there is a gage $\delta_{B}$ on $B$ such that

$$
\sum_{i=1}^{p}\left|f\left(y_{i}\right) \lambda\left(B_{i}\right)-F\left(B_{i}\right)\right|<\varepsilon
$$

for each $\delta_{B}$-fine $\varepsilon$-regular partition $\left\{\left(B_{1}, y_{1}\right), \ldots,\left(B_{p}, y_{p}\right)\right\}$ in $B$. With no loss of generality, we may assume that $\delta_{B}(x)=0$ for each $x \in \partial B$. Let $\varepsilon^{*}=(\beta / \alpha)^{m} \kappa \varepsilon$ and define a gage $\delta_{A}$ on $A$ by setting $\delta_{A}=\min \left\{\delta_{B} \circ \Phi / \beta, \Delta\right\}$. Choose a $\delta_{A}$-fine $\varepsilon^{*}$-regular partition $\left\{\left(A_{1}, x_{1}\right), \ldots,\left(A_{p}, x_{p}\right)\right\}$ in $A$. For $i=$ $1, \ldots, p$, let $K_{i}=\Phi\left(A_{i}\right)$ and $y_{i}=\Phi\left(x_{i}\right)$, and observe that $d\left(K_{i}\right)<\delta_{B}\left(y_{i}\right)$ and

$$
\frac{\lambda\left(K_{i}\right)}{d\left(K_{i}\right) \mathscr{H}\left(\partial K_{i}\right)} \geq\left(\frac{\alpha}{\beta}\right)^{m} r\left(A_{i}\right)>\kappa \varepsilon .
$$

It follows from Lemma 3.1 and Proposition 3.2 that each $K_{i}$ contains a figure $B_{i}$ such that

$$
r\left(B_{i}\right)>\varepsilon, \quad\left|f\left(y_{i}\right)\right| \cdot\left|\lambda\left(K_{i}\right)-\lambda\left(B_{i}\right)\right|<\varepsilon / p, \quad\left|G\left(A_{i}\right)-F\left(B_{i}\right)\right|<\varepsilon / p .
$$


As the figure $B_{i}$ may not contain the point $y_{i}$, an additional adjustment is necessary. Fix an integer $i$ with $1 \leq i \leq p$, and observe that $y_{i} \in B^{\circ}$ by the choice of $\delta_{B}$. Thus we can select nonoverlapping cubes $C_{1}, \ldots, C_{2^{m}}$ contained in $B$ whose common vertex is the point $y_{i}$. If $\left\{j_{1}, \ldots, j_{k}\right\}$ is the set of all indexes $j$ for which $x_{i} \in A_{j}$, then $1 \leq k \leq 2^{m}$ and we let

$$
B_{j_{s}}^{*}=\left[\left(B_{j_{s}} \cup C_{s}\right)-\bigcup_{r \neq s} C_{r}\right]^{-}
$$

for $s=1, \ldots, k$. Since $y_{i} \in K_{i}$, the cubes $C_{1}, \ldots, C_{2^{m}}$ can be chosen so small that $d\left(B_{i}^{*}\right)<\delta_{B}\left(y_{i}\right)$ and the above inequalities hold when $B_{i}$ is replaced by $B_{i}^{*}$. In particular, $\left\{\left(B_{1}^{*}, y_{1}\right), \ldots,\left(B_{p}^{*}, y_{p}\right)\right\}$ is a $\delta_{B}$-fine $\varepsilon$-regular partition in $B$, and we obtain

$$
\begin{aligned}
\sum_{i=1}^{p} \mid f \circ & \Phi\left(x_{i}\right) \cdot\left|\operatorname{det} \Phi\left(x_{i}\right)\right| \lambda\left(A_{i}\right)-G\left(A_{i}\right) \mid \\
\leq & \sum_{i=1}^{p}\left|f \circ \Phi\left(x_{i}\right)\right| \cdot|| \operatorname{det} \Phi\left(x_{i}\right)\left|\lambda\left(A_{i}\right)-\lambda\left(K_{i}\right)\right|+\sum_{i=1}^{p}\left|f\left(y_{i}\right)\right| \cdot\left|\lambda\left(K_{i}\right)-\lambda\left(B_{i}^{*}\right)\right| \\
& +\sum_{i=1}^{p}\left|f\left(y_{i}\right) \lambda\left(B_{i}^{*}\right)-F\left(B_{i}^{*}\right)\right|+\sum_{i=1}^{p}\left|F\left(B_{i}^{*}\right)-G\left(A_{i}\right)\right| \\
& <\sum_{i=1}^{p} \varepsilon \lambda\left(A_{i}\right)+p \frac{\varepsilon}{p}+\varepsilon+p \frac{\varepsilon}{p} \leq \varepsilon[\lambda(A)+3] .
\end{aligned}
$$

This proves the theorem.

\section{REFERENCES}

[Fa] K. J. Falconer, The geometry of fractal sets, Cambridge Univ. Press, Cambridge, 1985.

[Fe] H. Federer, Geometric measure theory, Springer-Verlag, New York, 1969.

[JK1] J. Jarnik and J. Kurzweil, A nonabsolutely convergent integral which admits transformation and can be used for integration on manifolds, Czechoslovak Math. J. 35 (1986), 116-139.

[JK2] $\frac{}{8-48}$. A new and more powerful concept of PU-integral, Czechoslovak Math. J. 38 (1988),

[KMP] J. Kurzweil, J. Mawhin, and W. F. Pfeffer, An integral defined by approximating $B V$ partitions of unity, Czechoslovak Math. J. 41 (1991), 695-712.

[P1] W. F. Pfeffer, The multidimensional fundamental theorem of calculus, J. Austral. Math. Soc. Ser. A 43 (1987), 143-170.

[P2] - The Gauss-Green theorem, Adv. in Math. 87 (1991), 93-147.

[P3] Lectures on geometric integration and the divergence theorem, Rend. Istit. Mat. Univ. Trieste (in press).

[R] W. Rudin, Real and complex analysis, McGraw-Hill, New York, 1987.

[Z] W. P. Ziemer, Weakly differentiable functions, Springer-Verlag, New York, 1989.

Department of Mathematics, Moscow State University, Moscow, Republic of Russia

Department of Mathematics, University of California, Davis, California 95616

E-mail address:

E-mail address: wfpfeffer@ucdavis.bitnet 